NoDEA

Nonlinear differ. equ. appl. 14 (2007) 377-390

1021-9722/07/040377-14

DOI $10.1007 / \mathrm{s} 00030-007-6005-0$ (c) Birkhäuser Verlag, Basel, 2007

Nonlinear Differential Equations

and Applications NoDEA

\title{
Local Lipschitz continuity of graphs with prescribed Levi mean curvature
}

\author{
Vittorio MARTINO \\ Dipartimento di Matematica \\ Università di Bologna \\ piazza di Porta S.Donato 5 \\ 40127 Bologna, Italy \\ e-mail:martino@dm.unibo.it \\ Annamaria MONTANARI \\ Dipartimento di Matematica \\ Università di Bologna \\ piazza di Porta S.Donato 5 \\ 40127 Bologna, Italy \\ e-mail:montanar@dm.unibo.it
}

\begin{abstract}
We prove interior gradient estimates of viscosity solutions of the prescribed Levi mean curvature equation.

2000 Mathematics Subject Classification: 35J70 and 35B45

Key words: Levi mean curvature and quasilinear degenerate elliptic pde's and local Lipschitz estimates.
\end{abstract}

\section{Introduction}

Starting from the existence results of Slodkowski Tomassini [17] and Debiard Gaveau [6], Citti Lanconelli and the second author in [5] proved the local smoothness of Lipschitz continuous graphs in $\mathbb{C}^{2}$, with prescribed smooth and non vanishing Levi curvature. However, these results left open the question of existence of Lipschitz continuous viscosity solutions under sharp conditions on the bound-

The second author was partially supported by Indam, within the interdisciplinary project "Nonlinear subelliptic equations of variational origin in contact geometry". 
ary data. In a recent work [14], by using the techniques of viscosity solutions, we proved the existence and uniqueness of continuous viscosity solution of the prescribed Levi mean curvature equation for every continuous boundary data. In this paper we give a sufficient condition on the prescribed Levi mean curvature which guarantees that such a solution is locally Lipschitz continuous.

Let us introduce some notations. For every $p=\left(p_{1}, \ldots, p_{N+1}\right) \in \mathbb{C}^{N+1}$ we denote by $p_{\bar{j}}=\bar{p}_{j}$ for every $j=1, \ldots, N+1$ and for every Hermitian matrix $r=\left(r_{i \bar{j}}\right)_{i, j=1}^{N+1}$ we define the Levi determinant (see [1])

$$
L_{i, j}(p, \bar{p}, r)=-\operatorname{det}\left(\begin{array}{ccc}
0 & p_{\bar{i}} & p_{\bar{j}} \\
p_{i} & r_{i \bar{i}} & r_{i \bar{j}} \\
p_{j} & r_{j \bar{i}} & r_{j \bar{j}}
\end{array}\right) .
$$

With these notations the prescribed Levi mean curvature equation of an oriented hypersurface with a defining function $f: \mathbb{C}^{N+1} \rightarrow \mathbb{R}$ and with outward unit normal $\nabla f /|\nabla f|$, writes as (see [15])

$$
k(z)=\frac{1}{N} \frac{1}{|\partial f|^{3}} L(\partial f, \bar{\partial} f, \partial \bar{\partial} f), \quad L(\partial f, \bar{\partial} f, \partial \bar{\partial} f)=\sum_{1 \leq i<j \leq N+1} L_{i, j}(\partial f, \bar{\partial} f, \partial \bar{\partial} f)
$$

where $k: \mathbb{C}^{N+1} \rightarrow \mathbb{R}$ is a prescribed function.

If the hypersurface is locally the graph of a function $u: \Omega \rightarrow \mathbb{R}$ with $\Omega \subset$ $\mathbb{R}^{n}, n=2 N+1$ then by identifying $z=\left(z_{1}, \ldots, z_{N+1}\right)=\left(x_{1}+i x_{N+1}, \ldots, x_{N}+\right.$ $\left.i x_{2 N}, x_{2 N+1}+i t\right)$ with $(x, t) \in \mathbb{R}^{n} \times \mathbb{R}$ and writing $f(z)=u(x)-t$, the prescribed Levi mean curvature equation (2) writes as

$$
k(x, u)=\frac{1}{N} \frac{2^{3}}{\left(1+|D u|^{2}\right)^{3 / 2}} F\left(D u, D^{2} u\right)
$$

where $D u$ and $D^{2} u$ are the Euclidean gradient and the Hessian matrix of $u$ respectively and $F$ is explicitly defined in (4). Let us stress that (3) is a quasilinear degenerate elliptic PDE (see Proposition 4). However, in spite of the lack of ellipticity of $F$ in one direction, in this paper we prove the following regularity result.

Theorem 1 Let $\Omega$ be a bounded open set in $\mathbb{R}^{n}$ and let $u \in C(\bar{\Omega})$ be a viscosity solution of (3) with $k \in C^{1}(\bar{\Omega} \times \mathbb{R})$ and non negative. If

$$
\frac{\partial k}{\partial u} \geq \alpha>0
$$

then $u$ is locally Lipschitz continuous and

$$
|D u(x)| \leq \frac{c}{d^{2}(x, \partial \Omega)}
$$


for a.e. $x \in \Omega$, where $d(\cdot, \partial \Omega)$ is the boundary distance and $c$ is a positive constant depending on the diameter of $\Omega$, on $M=\sup _{\bar{\Omega}}|u|, \alpha=\inf _{\bar{\Omega} \times[-M, M]} \frac{\partial k}{\partial u}, M_{1}=$ $\sup _{\bar{\Omega} \times[-M, M]} k$ and $M_{2}=\sup _{\bar{\Omega} \times[-\tilde{M}, \tilde{M}]}\left|\frac{\partial k}{\partial x}\right|$, with $\tilde{M}$ only depending on $M, M_{1}$.

The key tool in the proof of Theorem 1 is a surprising differential property of Levi determinants, which is proved in Section 2. Roughly speaking, this property allows us to apply the maximum principle to $|D u|^{2}$ times a cut-off function, because it permits to handle the remainder term. In Section 3 we prove Theorem 1 by using an approximation argument and uniform Lipschitz estimates.

The idea to use the maximum principle approach to get gradient estimates go back to Bernstein [2], [3]. Bernstein method was then developed by Ladyzhenskaya [10] and Ladyzhenskaya and Ural'tseva [11], [12] to yield both global and interior gradient estimates for uniformly elliptic equations. Later Serrin [16] extended these results to the prescribed classical mean curvature equation.

Let us stress that for the classical mean curvature equation an interior gradient bound holds without assumptions on the strictly monotonicity of the prescribed curvature function with respect to $u$. We recall that for the minimal surface equation an interior gradient bound with right hand side in exponential form was discovered, in the case of two variables, by Finn [8] and in the general case by Bombieri, De Giorgi and Miranda [4]. The method of the paper [4] depends upon an isoperimetric inequality of Federer and Fleming [7] and a resulting Sobolev inequality. This method was then used by Ladyzhenskaya and Ural'tseva in [13] to establish an interior gradient bounds for the general prescribed mean curvature equation.

For the prescribed mean Levi curvature equation (3) with $k=0$ we cannot expect that a similar interior gradient bound holds. Indeed, in the appendix we show that for every continuous function $U: A \rightarrow \mathbb{R}, A \subseteq \mathbb{R}, u(x)=U\left(x_{n}\right)$ is a viscosity solution of $F\left(D u, D^{2} u\right)=0$.

\section{A differential property of the Levi determinant}

In this section we differentiate the Levi determinant (1). We start with the case $N=1$

Proposition 1 If $N=1$ then

$$
\left|\frac{\partial L}{\partial p}\right|^{2}=\sum_{j, k, l=1}^{2} \frac{\partial L}{\partial r_{j \bar{k}}} r_{j \bar{l}} r_{l \bar{k}}
$$

where $\left|\frac{\partial L}{\partial p}\right|^{2}:=\sum_{j=1}^{N+1} \frac{\partial L}{\partial p_{j}} \frac{\partial L}{\partial p_{\bar{j}}}$ 
Proof. If $N=1$ then

$$
L(p, \bar{p}, r)=L_{1,2}(p, \bar{p}, r)=-\operatorname{det}\left(\begin{array}{lll}
0 & p_{\overline{1}} & p_{\overline{2}} \\
p_{1} & r_{1 \overline{1}} & r_{1 \overline{2}} \\
p_{2} & r_{2 \overline{1}} & r_{2 \overline{2}}
\end{array}\right)
$$

A direct computation shows that

$$
\begin{aligned}
\frac{\partial L}{\partial p_{1}} & =p_{\overline{1}} r_{2 \overline{2}}-p_{\overline{2}} r_{2 \overline{1}}, \quad \frac{\partial L}{\partial p_{2}}=p_{\overline{2}} r_{1 \overline{1}}-p_{\overline{1}} r_{1 \overline{2}} \\
\left|\frac{\partial L}{\partial p_{\overline{1}}}\right|^{2} & =p_{\overline{2}} p_{2} r_{2 \overline{1}} r_{1 \overline{2}}+p_{\overline{1}} p_{1} r_{2 \overline{2}} r_{2 \overline{2}}-p_{\overline{2}} p_{1} r_{2 \overline{1}} r_{2 \overline{2}}-p_{\overline{1}} p_{2} r_{2 \overline{2}} r_{1 \overline{2}} \\
\left|\frac{\partial L}{\partial p_{\overline{2}}}\right|^{2} & =p_{\overline{1}} p_{1} r_{1 \overline{2}} r_{2 \overline{1}}+p_{\overline{2}} p_{2} r_{1 \overline{1}} r_{1 \overline{1}}-p_{\overline{1}} p_{2} r_{1 \overline{2}} r_{1 \overline{1}}-p_{\overline{2}} p_{1} r_{1 \overline{1}} r_{2 \overline{1}} \\
\frac{\partial L}{\partial r_{1 \overline{1}}} & =p_{\overline{2}} p_{2}, \quad \frac{\partial L}{\partial r_{1 \overline{2}}}=-p_{\overline{1}} p_{2}, \\
\frac{\partial L}{\partial r_{2 \overline{1}}} & =-p_{\overline{2}} p_{1}, \quad \frac{\partial L}{\partial r_{2 \overline{2}}}=p_{\overline{1}} p_{1}
\end{aligned}
$$

and by substituting we get

$$
\begin{aligned}
\left|\frac{\partial L}{\partial p}\right|^{2}= & +p_{\overline{2}} p_{2}\left(r_{1 \overline{1}} r_{1 \overline{1}}+r_{1 \overline{2}} r_{2 \overline{1}}\right)-p_{\overline{1}} p_{2}\left(r_{1 \overline{1}} r_{1 \overline{2}}+r_{1 \overline{2}} r_{2 \overline{2}}\right) \\
& -p_{\overline{2}} p_{1}\left(r_{2 \overline{1}} r_{1 \overline{1}}+r_{2 \overline{2}} r_{2 \overline{1}}\right)+p_{\overline{1}} p_{1}\left(r_{2 \overline{1}} r_{1 \overline{2}}+r_{2 \overline{2}} r_{2 \overline{2}}\right) \\
= & +\frac{\partial L}{\partial r_{1 \overline{1}}}\left(r_{1 \overline{1}} r_{1 \overline{1}}+r_{1 \overline{2}} r_{2 \overline{1}}\right)+\frac{\partial L}{\partial r_{1 \overline{2}}}\left(r_{1 \overline{1}} r_{1 \overline{2}}+r_{1 \overline{2}} r_{2 \overline{2}}\right) \\
& +\frac{\partial L}{\partial r_{2 \overline{1}}}\left(r_{2 \overline{1}} r_{1 \overline{1}}+r_{2 \overline{2}} r_{2 \overline{1}}\right)+\frac{\partial L}{\partial r_{2 \overline{2}}}\left(r_{2 \overline{1}} r_{1 \overline{2}}+r_{2 \overline{2}} r_{2 \overline{2}}\right) \\
= & \sum_{j, k, l=1}^{2} \frac{\partial L}{\partial r_{j \bar{k}}} r_{j \bar{l}} r_{l \bar{k}}
\end{aligned}
$$

We now use Proposition 1 to handle the general case $N \in I N$.

Proposition 2 For all $N \in I N$ we have

$$
\left|\frac{\partial L}{\partial p}\right|^{2} \leq N \sum_{m, q, t=1}^{N+1} \frac{\partial L}{\partial r_{m \bar{q}}} r_{m \bar{t}} r_{t \bar{q}}
$$

Proof. Since $L(p, \bar{p}, r)=\sum_{1 \leq i<j \leq N+1} L_{i, j}(p, \bar{p}, r)$ and for all $1 \leq \ell \leq N+1, L_{i, j}$ depends on $p_{\ell}$ iff $i=\ell$ or $j=\ell$, we have

$$
\frac{\partial L}{\partial p_{\ell}}=\sum_{1 \leq i<\ell} \frac{\partial L_{i, \ell}}{\partial p_{\ell}}+\sum_{\ell<j \leq N+1} \frac{\partial L_{\ell, j}}{\partial p_{\ell}}
$$


and by the triangle inequality

$$
\left|\frac{\partial L}{\partial p_{\ell}}\right| \leq \sum_{1 \leq i<\ell}\left|\frac{\partial L_{i, \ell}}{\partial p_{\ell}}\right|+\sum_{\ell<j \leq N+1}\left|\frac{\partial L_{\ell, j}}{\partial p_{\ell}}\right| .
$$

By Proposition 1 we have

$$
\begin{aligned}
\left|\frac{\partial L}{\partial p}\right|^{2}=\sum_{\ell=1}^{N+1}\left|\frac{\partial L}{\partial p_{\ell}}\right|^{2} & \leq \sum_{\ell=1}^{N+1}\left(\sum_{1 \leq i<\ell}\left|\frac{\partial L_{i, \ell}}{\partial p_{\ell}}\right|^{N} \sum_{\ell<j \leq N+1}\left|\frac{\partial L_{\ell, j}}{\partial p_{\ell}}\right|\right)^{2} \\
& \leq N \sum_{\ell=1}^{N+1}\left(\sum_{1 \leq i<\ell}\left|\frac{\partial L_{i, \ell}}{\partial p_{\ell}}\right|^{2}+\sum_{\ell<j \leq N+1}\left|\frac{\partial L_{\ell, j}}{\partial p_{\ell}}\right|^{2}\right) \\
& =N \sum_{i=1}^{N+1} \sum_{\ell \neq i}\left(\left|\frac{\partial L_{i, \ell}}{\partial p_{\ell}}\right|^{2}+\left|\frac{\partial L_{i, \ell}}{\partial p_{i}}\right|^{2}\right) \\
& =N \sum_{i=1}^{N+1} \sum_{\ell \neq i} \sum_{m, q, t \in\{i, \ell\}} \frac{\partial L_{i, \ell}}{\partial r_{m \bar{q}}} r_{m \bar{t}} r_{t \bar{q}} \\
& =N \sum_{m, q, t=1}^{N+1} \frac{\partial L}{\partial r_{m \bar{q}}} r_{m \bar{t}} r_{t \bar{q}}
\end{aligned}
$$

Corollary 1 The Hermitian matrix $\left(\frac{\partial L}{\partial r_{m \bar{q}}}\right)_{m, q=1}^{N+1}$ is non negative definite.

Proof. For every $\zeta \in \mathbb{C}^{N+1}$ we have

$$
\begin{aligned}
& \sum_{m, q=1}^{N+1} \frac{\partial L}{\partial r_{m \bar{q}}} \zeta_{q} \bar{\zeta}_{m}=\sum_{m, q=1}^{N+1}\left(\sum_{1 \leq i<j \leq N+1} \frac{\partial L_{i, j}}{\partial r_{m \bar{q}}}\right) \zeta_{q} \bar{\zeta}_{m} \\
& \quad=\sum_{m, q=1}^{N+1}\left(\sum_{1 \leq i<j \leq N+1}\left(\left|p_{j}\right|^{2} \delta_{i m} \delta_{i q}+\left|p_{i}\right|^{2} \delta_{j m} \delta_{j q}-p_{i} p_{\bar{j}} \delta_{m j} \delta_{i q}-p_{j} p_{\bar{i}} \delta_{m i} \delta_{j q}\right)\right) \zeta_{q} \bar{\zeta}_{m} \\
& =\sum_{1 \leq i<j \leq N+1}\left(\left|p_{j}\right|^{2} \zeta_{i} \bar{\zeta}_{i}+\left|p_{i}\right|^{2} \zeta_{j} \bar{\zeta}_{j}-p_{i} p_{\bar{j}} \zeta_{i} \bar{\zeta}_{j}-p_{j} p_{\bar{i}} \zeta_{j} \bar{\zeta}_{i}\right) \\
& =\sum_{1 \leq i<j \leq N+1}\left|p_{j} \bar{\zeta}_{i}-p_{i} \bar{\zeta}_{j}\right|^{2} \geq 0
\end{aligned}
$$

For the function $F$ in (4) similar properties hold. We first explicitly write the definition of $F$ in terms of $L$ and of a change of variable. For every $\xi \in \mathbb{R}^{n}$ and for every $n \times n$ symmetric matrix $X=\left(X_{i j}\right)_{i, j=1}^{n}$

$$
F(\xi, X)=L(p, \bar{p}, r)
$$


where for $1 \leq j \leq N$

$$
p_{j}=\frac{1}{2}\left(\xi_{j}-i \xi_{N+j}\right), \quad p_{N+1}=\frac{1}{2}\left(\xi_{2 N+1}+i\right),
$$

and the matrix $r$ is

$$
r=\frac{1}{4} \bar{J}^{T} X J, \quad \text { with } J=\left(\begin{array}{ll}
I_{N} & 0 \\
i I_{N} & 0 \\
0 & 1
\end{array}\right)
$$

Proposition 3 Let $F$ be the function in (4). Then

$$
\left|\frac{\partial F}{\partial \xi}\right|^{2} \leq \frac{N}{2} \sum_{i, j, k=1}^{n} \frac{\partial F}{\partial X_{i j}} X_{i k} X_{k j}
$$

with $n=2 N+1$

Proof. By the change of variable (5) we have for $1 \leq j \leq N$

$$
\begin{aligned}
\frac{\partial F}{\partial \xi_{j}} & =\frac{1}{2}\left(\frac{\partial L}{\partial p_{j}}+\frac{\partial L}{\partial \bar{p}_{j}}\right)=\operatorname{Re} \frac{\partial L}{\partial p_{j}} \\
\frac{\partial F}{\partial \xi_{j+N}} & =-\frac{i}{2}\left(\frac{\partial L}{\partial p_{j}}-\frac{\partial L}{\partial \bar{p}_{j}}\right)=\operatorname{Im} \frac{\partial L}{\partial p_{j}} \\
\frac{\partial F}{\partial \xi_{n}} & =\frac{1}{2}\left(\frac{\partial L}{\partial p_{N}}+\frac{\partial L}{\partial \bar{p}_{N}}\right)=\operatorname{Re} \frac{\partial L}{\partial p_{N}}
\end{aligned}
$$

Hence

$$
\left|\frac{\partial F}{\partial \xi}\right|^{2}=\sum_{j=1}^{n}\left(\frac{\partial F}{\partial \xi_{j}}\right)^{2}=\sum_{j=1}^{N}\left|\frac{\partial L}{\partial p_{j}}\right|^{2}+\left(R e \frac{\partial L}{\partial p_{N}}\right)^{2} \leq\left|\frac{\partial L}{\partial p}\right|^{2}
$$

and by Proposition 2 and the change of variable (6)

$$
\begin{aligned}
\left|\frac{\partial L}{\partial p}\right|^{2} & \leq N \sum_{m, q, t=1}^{N+1} \frac{\partial L}{\partial r_{m \bar{q}}} r_{m \bar{t}} r_{t \bar{q}}=N \operatorname{Tr}\left(\left(\frac{\partial L}{\partial \bar{r}}\right)^{T} r r\right) \\
& =\frac{N}{16} \operatorname{Tr}\left(\left(\frac{\partial L}{\partial \bar{r}}\right)^{T} \bar{J}^{T} X J \bar{J}^{T} X J\right)
\end{aligned}
$$

Since $X J\left(\frac{\partial L}{\partial \bar{r}}\right)^{T} \bar{J}^{T} X$ is a non negative definite Hermitian matrix and

$$
0 \leq J \bar{J}^{T}=\left(\begin{array}{ccc}
I_{N} & -i I_{N} & 0 \\
i I_{N} & I_{N} & 0 \\
0 & 0 & 1
\end{array}\right) \leq 2 I_{n}
$$


we have

$$
\begin{aligned}
\left|\frac{\partial F}{\partial \xi}\right|^{2} & \leq\left|\frac{\partial L}{\partial p}\right|^{2} \leq \frac{N}{16} \operatorname{Tr}\left(X J\left(\frac{\partial L}{\partial \bar{r}}\right)^{T} \bar{J}^{T} X J \bar{J}^{T}\right) \\
& \leq \frac{N}{8} \operatorname{Tr}\left(X J\left(\frac{\partial L}{\partial \bar{r}}\right)^{T} \bar{J}^{T} X\right) .
\end{aligned}
$$

We now use again (6) to estimate the righthand side in (7). We have

$$
\frac{\partial F}{\partial X_{i j}}=\sum_{l, p=1}^{N+1} \frac{\partial L}{\partial r_{l \bar{p}}} \frac{\partial r_{l \bar{p}}}{\partial X_{i j}}=\frac{1}{4} \sum_{l, p=1}^{N+1} \frac{\partial L}{\partial r_{l \bar{p}}} \bar{J}_{l i}^{T} J_{j p}
$$

and

$$
\begin{aligned}
\operatorname{Tr}\left(\left(\frac{\partial F}{\partial X}\right)^{T} X X\right) & =\sum_{i, j, k=1}^{n} \frac{\partial F}{\partial X_{i j}} X_{i k} X_{k j}=\frac{1}{4} \sum_{i, j, k}^{n}\left(\sum_{l, p=1}^{N+1} \frac{\partial L}{\partial r_{l \bar{p}}} \bar{J}_{l i}^{T} J_{j p} X_{i k} X_{k j}\right) \\
& =\frac{1}{4} \sum_{i, j, k}^{n}\left(\sum_{l, p=1}^{N+1} X_{k j} J_{j p} \frac{\partial L}{\partial r_{l \bar{p}}} \bar{J}_{l i}^{T} X_{i k}\right) \\
& =\frac{1}{4} \operatorname{Tr}\left(X J\left(\frac{\partial L}{\partial \bar{r}}\right)^{T} \bar{J}^{T} X\right) .
\end{aligned}
$$

By substituting (9) in (7) we finally obtain

$$
\left|\frac{\partial F}{\partial \xi}\right|^{2} \leq \frac{N}{2} \sum_{i, j, k=1}^{n} \frac{\partial F}{\partial X_{i j}} X_{i k} X_{k j} .
$$

Proposition 4 The matrix $\left(\frac{\partial F}{\partial X_{i j}}\right)_{i, j=1}^{n}$ is non negative definite and

$$
\sum_{j=1}^{n} \frac{\partial F}{\partial X_{j j}}(\xi, X) \leq \frac{N}{8}\left(1+|\xi|^{2}\right)
$$

Proof. The first assertion follows by (8) and by Corollary 1. Moreover, by (6) and (5)

$$
\begin{aligned}
\sum_{j=1}^{n} \frac{\partial F}{\partial X_{j j}} & =\frac{1}{4} \sum_{j=1}^{n} \sum_{l, q=1}^{N+1} \frac{\partial L}{\partial r_{l \bar{q}}} \bar{J}_{l j}^{T} J_{j q}=\frac{1}{2} \sum_{l=1}^{N} \frac{\partial L}{\partial r_{l \bar{l}}}+\frac{1}{4} \frac{\partial L}{\partial r_{N+1 \overline{N+1}}} \\
& =\frac{1}{2} \sum_{l=1}^{N}\left(|p|^{2}-\left|p_{l}\right|^{2}\right)+\frac{1}{4}\left(\left(|p|^{2}-\left|p_{N+1}\right|^{2}\right)\right) \leq \frac{N}{2}|p|^{2}=\frac{N}{8}\left(|\xi|^{2}+1\right)
\end{aligned}
$$




\section{Interior gradient estimate}

In this section we shall prove Theorem 1 by using an approximation argument and uniform a priori estimates.

We denote by $x$ a point in $\mathbb{R}^{n}$ and by $\mathcal{S}(n)$ the space of all $n \times n$ symmetric matrices. For every $(\xi, X) \in \mathbb{R}^{n} \times \mathcal{S}(n)$ and $\varepsilon>0$ we define

$$
F^{\varepsilon}(\xi, X)=F(\xi, X)+\varepsilon \operatorname{Tr} X,
$$

with $F$ as in (4).

Proof of Theorem 1. If $u \in C(\bar{\Omega})$ is a viscosity solution of (3) with $k \in C^{1}(\Omega \times \mathbb{R})$, then for every ball $B \subset \subset \Omega$ we consider a solution $u^{\varepsilon} \in C^{3}(B)$ of the elliptic PDE

$$
F^{\varepsilon}\left(D u^{\varepsilon}, D^{2} u^{\varepsilon}\right)=\frac{N}{2^{3}}\left(1+\left|D u^{\varepsilon}\right|^{2}\right)^{3 / 2} k\left(x, u^{\varepsilon}\right)
$$

with boundary data $u$ on $\partial B$. Let us recall that the existence of a solution $u^{\varepsilon} \in$ $C^{2}(B)$ of (10) for every continuous boundary data is guaranteed by $[9$, Theorem 15.18]. The $C^{3}$ regularity follows by the uniform ellipticity of $F^{\varepsilon}$ for every $\varepsilon>0$. Moreover, if $M=\sup _{\bar{\Omega}}|u|$ and $k$ is non negative then $F^{\varepsilon}\left(D M, D^{2} M\right)=0 \leq$ $F^{\varepsilon}\left(D u^{\varepsilon}, D^{2} u^{\varepsilon}\right)$ and by the comparison principle $\sup _{B} u^{\varepsilon} \leq M$. By [14, estimate $(4.2)]$ we also have

$$
\sup _{B}\left|u^{\varepsilon}\right| \leq \tilde{M}=M+C,
$$

with $C$ only depending on $\sup _{\bar{\Omega} \times[-M, M]} k$. We shall prove that if inf $\frac{\partial k}{\partial u}=\alpha>0$ then $w:=\frac{\left|D u^{\varepsilon}\right|^{2}}{2}$ is locally bounded by a constant independent of $\varepsilon$. For $\varepsilon \rightarrow 0$, $u^{\varepsilon}$ will uniformly converge to a Lipschitz continuous viscosity solution $\tilde{u}$ of (3), which agrees with $u$ on $\partial B$. The comparison principle in [14] will guarantee that $\tilde{u}=u$ is Lipschitz continuous on $B$ and the thesis of Theorem 1 will follow.

We start by differentiating the equality (10) with respect to $x_{l}$. For a sake of simplicity in the sequel we shall denote by $u_{j}=\frac{\partial u}{\partial x_{j}}, u_{i j}=\frac{\partial^{2} u}{\partial x_{j} \partial x_{i}}$, and use a similar notation for third order partial derivatives. We get

$$
\begin{aligned}
\sum_{i, j=1}^{n} \frac{\partial F^{\varepsilon}}{\partial X_{i j}} u_{i j l}^{\varepsilon}+\sum_{i=1}^{n} \frac{\partial F^{\varepsilon}}{\partial \zeta_{i}} u_{i l}^{\varepsilon}= & \frac{3 N}{2^{3}}(2 w+1)^{\frac{1}{2}} \sum_{i=1}^{n} u_{i}^{\varepsilon} u_{i l}^{\varepsilon} k\left(x, u^{\varepsilon}\right) \\
& +\frac{N}{2^{3}}(2 w+1)^{\frac{3}{2}}\left(\frac{\partial k}{\partial x_{l}}+\frac{\partial k}{\partial u} u_{l}^{\varepsilon}\right)
\end{aligned}
$$

By multiplying (12) by $u_{l}^{\varepsilon}$ and summing up in $l$ we get

$$
\begin{aligned}
\sum_{i, j, l=1}^{n} \frac{\partial F^{\varepsilon}}{\partial X_{i j}} u_{i j l}^{\varepsilon} u_{l}^{\varepsilon}+\sum_{i, l=1}^{n} \frac{\partial F^{\varepsilon}}{\partial \zeta_{i}} u_{i l}^{\varepsilon} u_{l}^{\varepsilon}= & \frac{3 N}{2^{3}}(2 w+1)^{\frac{1}{2}} k\left(x, u^{\varepsilon}\right) \sum_{i, l=1}^{n} u_{i}^{\varepsilon} u_{i l}^{\varepsilon} u_{l}^{\varepsilon} \\
& +\frac{N}{2^{3}}(2 w+1)^{\frac{3}{2}}\left(2 \frac{\partial k}{\partial u} w+\sum_{l=1}^{n} \frac{\partial k}{\partial x_{l}} u_{l}^{\varepsilon}\right)
\end{aligned}
$$


Since $w_{i}=\sum_{l=1}^{n} u_{l}^{\varepsilon} u_{i l}^{\varepsilon}$ and $w_{i j}=\sum_{l=1}^{n}\left(u_{l j}^{\varepsilon} u_{i l}^{\varepsilon}+u_{l}^{\varepsilon} u_{i j l}^{\varepsilon}\right)$ we can rewrite equation (13) as

$$
\begin{aligned}
\sum_{i, j, l=1}^{n} \frac{\partial F^{\varepsilon}}{\partial X_{i j}}\left(w_{i j}-u_{i l}^{\varepsilon} u_{l j}^{\varepsilon}\right)+\sum_{i, l=1}^{n} \frac{\partial F^{\varepsilon}}{\partial \zeta_{i}} w_{i}= & \frac{3 N}{2^{3}}(2 w+1)^{\frac{1}{2}} k\left(x, u^{\varepsilon}\right) \sum_{i}^{n} u_{i}^{\varepsilon} w_{i} \\
& +\frac{N}{2^{3}}(2 w+1)^{\frac{3}{2}}\left(2 \frac{\partial k}{\partial u} w+\sum_{l=1}^{n} \frac{\partial k}{\partial x_{l}} u_{l}^{\varepsilon}\right)
\end{aligned}
$$

Let $\mathcal{L}$ be the following second order elliptic operator

$$
\mathcal{L}=\sum_{i, j=1}^{n} \frac{\partial F^{\varepsilon}}{\partial X_{i j}} \partial_{i j}+\sum_{i=1}^{n} \frac{\partial F^{\varepsilon}}{\partial \zeta_{i}} \partial_{i}-\frac{3 N}{2^{3}}(2 w+1)^{\frac{1}{2}} k\left(x, u^{\varepsilon}\right) \sum_{i=1}^{n} u_{i}^{\varepsilon} \partial_{i} .
$$

Then equation (14) writes as

$$
\mathcal{L}(w)=\sum_{i, j, l=1}^{n} \frac{\partial F^{\varepsilon}}{\partial X_{i j}} u_{i l}^{\varepsilon} u_{l j}^{\varepsilon}+\frac{N}{2^{3}}(2 w+1)^{\frac{3}{2}}\left(2 \frac{\partial k}{\partial u} w+\sum_{l=1}^{n} \frac{\partial k}{\partial x_{l}} u_{l}^{\varepsilon}\right)
$$

For every $x \in \Omega$ let $R>0$ such that $B=B(x, R) \subseteq \Omega$ and let $\varphi \in C^{2}(\bar{B})$ such that $0 \leq \varphi \leq 1$ in $\bar{B}, \varphi=0$ on $\partial B$ and $\varphi>0$ in $B$. We shall choose such a function below. We shall apply $\mathcal{L}$ to the product $v=w \varphi$. We have

$$
\begin{aligned}
v_{i} & =w_{i} \varphi+w \varphi_{i} \\
v_{i j} & =w_{i j} \varphi+w_{i} \varphi_{j}+w_{j} \varphi_{i}+w \varphi_{i j}
\end{aligned}
$$

We now apply $\mathcal{L}$ to $v$. By remarking that $\frac{\partial F^{\varepsilon}}{\partial X_{i j}}$ is a symmetric matrix we get

$$
\begin{aligned}
\mathcal{L}(v) & =\mathcal{L}(w) \varphi+w \mathcal{L}(\varphi)+2 \sum_{i, j=1}^{n} \frac{\partial F^{\varepsilon}}{\partial X_{i j}} \varphi_{j} w_{i} \\
& =\mathcal{L}(w) \varphi+w \mathcal{L}(\varphi)+2 \sum_{i, j=1}^{n} \frac{\partial F^{\varepsilon}}{\partial X_{i j}} \varphi_{j} \frac{\left(v_{i}-w \varphi_{i}\right)}{\varphi}
\end{aligned}
$$

We now consider the elliptic PDO

$$
H=\mathcal{L}-2 \sum_{i, j=1}^{n} \frac{\partial F^{\varepsilon}}{\partial X_{i j}} \frac{\varphi_{j}}{\varphi} \partial_{i}
$$

and define

$$
\begin{aligned}
E & =\sum_{i, j, l=1}^{n} \frac{\partial F^{\varepsilon}}{\partial X_{i j}} u_{i l}^{\varepsilon} u_{l j}^{\varepsilon}=\sum_{i, j, l=1}^{n} \frac{\partial F}{\partial X_{i j}} u_{i l}^{\varepsilon} u_{l j}^{\varepsilon}+\varepsilon \sum_{i, l=1}^{n} u_{i l}^{\varepsilon} u_{l i}^{\varepsilon} \\
& \geq \sum_{i, j, l=1}^{n} \frac{\partial F}{\partial X_{i j}} u_{i l}^{\varepsilon} u_{l j}^{\varepsilon} .
\end{aligned}
$$


We have

$$
\begin{aligned}
H(v)= & \varphi\left(E+\frac{N}{2^{3}}(2 w+1)^{\frac{3}{2}}\left(2 \frac{\partial k}{\partial u} w+\sum_{l=1}^{n} \frac{\partial k}{\partial x_{l}} u_{l}^{\varepsilon}\right)\right) \\
& +\left(\mathcal{L}(\varphi)-2 \sum_{i, j=1}^{n} \frac{\partial F^{\varepsilon}}{\partial X_{i j}} \frac{\varphi_{i} \varphi_{j}}{\varphi}\right) w
\end{aligned}
$$

Let $x$ be a local maximum point for $v$, then $H(v)(x) \leq 0$ and at $x$

$0 \geq \varphi\left(E+\frac{N}{2^{3}}(2 w+1)^{\frac{3}{2}}\left(2 \frac{\partial k}{\partial u} w+\sum_{l=1}^{n} \frac{\partial k}{\partial x_{l}} u_{l}^{\varepsilon}\right)\right)+\left(\mathcal{L}(\varphi)-2 \sum_{i, j=1}^{n} \frac{\partial F^{\varepsilon}}{\partial X_{i j}} \frac{\varphi_{i} \varphi_{j}}{\varphi}\right) w$

Since

$$
\mathcal{L}(\varphi)=\sum_{i, j=1}^{n} \frac{\partial F^{\varepsilon}}{\partial X_{i j}} \varphi_{i j}+\sum_{i=1}^{n} \frac{\partial F^{\varepsilon}}{\partial \zeta_{i}} \varphi_{i}-\frac{3 N}{2^{3}}(2 w+1)^{\frac{1}{2}} k\left(x, u^{\varepsilon}\right) \sum_{i, l=1}^{n} u_{i}^{\varepsilon} \varphi_{i}
$$

and by Proposition 3

$$
\sum_{i=1}^{n} \frac{\partial F^{\varepsilon}}{\partial \zeta_{i}} \varphi_{i} \geq-|D \varphi|\left|\frac{\partial F^{\varepsilon}}{\partial \zeta}\right| \geq-\frac{\sqrt{N}}{\sqrt{2}}|D \varphi| \sqrt{E}
$$

at $x$ we have the inequality

$$
\begin{aligned}
0 \geq & \varphi E-\frac{\sqrt{N}}{\sqrt{2}}|D \varphi| \sqrt{E} w+\varphi\left(\frac{N}{2^{3}}(2 w+1)^{\frac{3}{2}}\left(2 \frac{\partial k}{\partial u} w+\sum_{l=1}^{n} \frac{\partial k}{\partial x_{l}} u_{l}^{\varepsilon}\right)\right) \\
& +w\left(\sum_{i, j=1}^{n} \frac{\partial F^{\varepsilon}}{\partial X_{i j}}\left(\varphi_{i j}-2 \frac{\varphi_{i} \varphi_{j}}{\varphi}\right)-\frac{3 N}{2^{3}}(2 w+1)^{\frac{1}{2}} k\left(x, u^{\varepsilon}\right) \sum_{i, l=1}^{n} u_{i}^{\varepsilon} \varphi_{i}\right) \\
\geq & -\frac{N w^{2}|D \varphi|^{2}}{8 \varphi}+\varphi\left(\frac{N}{2^{3}}(2 w+1)^{\frac{3}{2}}\left(2 \frac{\partial k}{\partial u} w+\sum_{l=1}^{n} \frac{\partial k}{\partial x_{l}} u_{l}^{\varepsilon}\right)\right) \\
& +w\left(\sum_{i, j=1}^{n} \frac{\partial F^{\varepsilon}}{\partial X_{i j}}\left(\varphi_{i j}-2 \frac{\varphi_{i} \varphi_{j}}{\varphi}\right)-\frac{3 N}{2^{3}}(2 w+1)^{\frac{1}{2}} k\left(x, u^{\varepsilon}\right) \sum_{i, l=1}^{n} u_{i}^{\varepsilon} \varphi_{i}\right)
\end{aligned}
$$


i.e.

$$
\begin{aligned}
\frac{N}{4} \varphi & (2 w+1)^{\frac{3}{2}} \frac{\partial k}{\partial u} w \\
\leq & \frac{N w^{2}|D \varphi|^{2}}{8 \varphi}-\frac{N}{2^{3}} \varphi(2 w+1)^{\frac{3}{2}} \sum_{l=1}^{n} \frac{\partial k}{\partial x_{l}} u_{l}^{\varepsilon} \\
& +\frac{N}{2^{3}} w(2 w+1)^{\frac{1}{2}} k\left(x, u^{\varepsilon}\right) \sum_{i, l=1}^{n} u_{i}^{\varepsilon} \varphi_{i}+w \sum_{i, j=1}^{n} \frac{\partial F^{\varepsilon}}{\partial X_{i j}}\left(2 \frac{\varphi_{i} \varphi_{j}}{\varphi}-\varphi_{i j}\right) \\
\leq & \frac{N}{2^{3}}\left(\frac{w^{2}|D \varphi|^{2}}{\varphi}+\varphi(2 w+1)^{\frac{3}{2}} \sqrt{w} M_{2}+w(2 w+1)^{\frac{1}{2}} M_{1}|D \varphi| \sqrt{w}\right) \\
& +w \sum_{i, j=1}^{n} \frac{\partial F^{\varepsilon}}{\partial X_{i j}}\left(2 \frac{\varphi_{i} \varphi_{j}}{\varphi}-\varphi_{i j}\right)
\end{aligned}
$$

where $M_{1}=\sqrt{2} \max _{\Omega \times[-M, M]}|k|, M_{2}=\sqrt{2} \max _{\Omega \times[-\tilde{M}, \tilde{M}]}\left|\partial_{x} k\right|$, with $M=\max _{\bar{\Omega}}|u|$ and $\tilde{M}$ as in (11). Since $0<\alpha \leq \frac{\partial k}{\partial u}$ we can divide equation (15) by $\frac{N}{4} \sqrt{\varphi}(2 w+$ $1)^{\frac{3}{2}} \alpha \sqrt{w}$ and we get

$$
\begin{aligned}
\sqrt{\varphi w} & \leq C_{1} \frac{|D \varphi|^{2}}{\varphi^{\frac{3}{2}}}+C_{2} \varphi^{\frac{1}{2}}+C_{3} \frac{|D \varphi|}{\varphi^{\frac{1}{2}}}+C_{4}(2 w+1)^{-1} \sum_{i, j=1}^{n} \frac{\partial F^{\varepsilon}}{\partial X_{i j}}\left(2 \frac{\varphi_{i} \varphi_{j}}{\varphi^{\frac{3}{2}}}-\frac{\varphi_{i j}}{\varphi^{\frac{1}{2}}}\right) \\
& \leq C_{1} \frac{|D \varphi|^{2}}{\varphi^{\frac{3}{2}}}+C_{2} \varphi^{\frac{1}{2}}+C_{3} \frac{|D \varphi|}{\varphi^{\frac{1}{2}}}+C_{4} \frac{\sqrt{w}}{(2 w+1)^{3 / 2}} \sum_{i, j=1}^{n} \frac{\partial F^{\varepsilon}}{\partial X_{i j}}\left(2 \frac{\varphi_{i} \varphi_{j}}{\varphi^{\frac{3}{2}}}-\frac{\varphi_{i j}}{\varphi^{\frac{1}{2}}}\right)
\end{aligned}
$$

where $C_{1}, C_{2}, C_{3}, C_{4}$ are non negative constants depending on $\alpha, M_{1}, M_{2}$.

We now choose $\varphi=\left(R^{2}-|x|^{2}\right)^{4}$ and compute its derivatives

$$
\begin{aligned}
\varphi_{i} & =-8\left(R^{2}-|x|^{2}\right)^{3} x_{i} \\
|D \varphi| & =8\left(R^{2}-|x|^{2}\right)^{3}|x| \\
\varphi_{i j} & =48\left(R^{2}-|x|^{2}\right)^{2} x_{j} x_{i}-8\left(R^{2}-|x|^{2}\right)^{3} \delta_{i j}
\end{aligned}
$$

and we have the following estimates

$$
\begin{aligned}
\frac{|D \varphi|^{2}}{\varphi^{\frac{3}{2}}} & =\frac{64\left(R^{2}-|x|^{2}\right)^{6}|x|^{2}}{\left(R^{2}-|x|^{2}\right)^{6}} \leq 64 R^{2} \\
\varphi^{\frac{1}{2}} & =\left(R^{2}-|x|^{2}\right)^{2} \leq R^{4} \\
\frac{|D \varphi|}{\varphi^{\frac{1}{2}}} & =\frac{8\left(R^{2}-|x|^{2}\right)^{3}|x|}{\left(R^{2}-|x|^{2}\right)^{2}} \leq 8 R^{3} \\
2 \frac{\varphi_{i} \varphi_{j}}{\varphi^{\frac{3}{2}}}-\frac{\varphi_{i j}}{\varphi^{\frac{1}{2}}} & =128 \frac{\left(R^{2}-|x|^{2}\right)^{6} x_{i} x_{j}}{\left(R^{2}-|x|^{2}\right)^{6}}-\frac{48\left(R^{2}-|x|^{2}\right)^{2} x_{j} x_{i}-8\left(R^{2}-|x|^{2}\right)^{3} \delta_{i j}}{\left(R^{2}-|x|^{2}\right)^{2}} \\
& =80 x_{i} x_{j}+8\left(R^{2}-|x|^{2}\right) \delta_{i j} \leq 88 R^{2} \delta_{i j}
\end{aligned}
$$


Since the symmetric matrix $\frac{\partial F^{\varepsilon}}{\partial X_{i j}}$ is non negative definite and by Proposition 4 with $|\xi|^{2}=2 w$

$$
\sum_{j=1}^{n} \frac{\partial F^{\varepsilon}}{\partial X_{j j}}=\sum_{j=1}^{n} \frac{\partial F}{\partial X_{j j}}+n \varepsilon \leq \frac{N}{8}(2 w+1)+n \varepsilon
$$

by redefining constants we get

$$
\sqrt{v}=\sqrt{\varphi w} \leq C_{1} R^{2}+C_{2} R^{4}+C_{3} R^{3}+C_{4} R^{2} \leq C_{0}
$$

where $C_{0}$ depends on $\alpha, M_{1}, M_{2}$ and on the diameter of the bounded set $\Omega$. Since $x$ is a local maximum of $v$, for every $x_{0} \in B(x, R)$ we have $v\left(x_{0}\right) \leq v(x)$ and by (17)

$$
\sqrt{\varphi\left(x_{0}\right) w\left(x_{0}\right)} \leq \sqrt{\varphi(x) w(x)} \leq C_{0} .
$$

Hence

$$
\frac{\left|D u^{\varepsilon}\left(x_{0}\right)\right|}{\sqrt{2}} \leq \frac{C_{0}}{\sqrt{\varphi\left(x_{0}\right)}}=\frac{C_{0}}{\left(R^{2}-\left|x_{0}\right|^{2}\right)^{2}}=\frac{C_{0}}{\left(R-\left|x_{0}\right|\right)^{2}\left(R+\left|x_{0}\right|\right)^{2}}
$$

and we can conclude that

$$
\left|D u^{\varepsilon}\left(x_{0}\right)\right| \leq \frac{C}{d^{2}\left(x_{0}, \partial B\right)}
$$

where $d(\cdot, \partial B)$ is the boundary distance.

\section{Appendix}

In this section we show that, for every continuous function $U: A \rightarrow \mathbb{R}, A \subseteq$ $\mathbb{R}$, the function $u(x)=u\left(x_{1}, \ldots, x_{2 N}, x_{n}\right)=U\left(x_{n}\right)$ is a viscosity solution of $F\left(D u, D^{2} u\right)=0$ in $\Omega=\operatorname{int}(A \times B)$, for every $B \subseteq \mathbb{R}^{2 N}$.

For every $x \in \Omega$ and $\phi \in C^{2}(\Omega)$ such that $u-\phi$ has a local maximum at $x$, we have $\phi_{j}(x)=u_{j}(x)=0$ for every $j=1, \ldots, 2 N$, and for every $\eta \in \mathbb{R}^{2 N}$

$$
0=\sum_{i, j=1}^{2 N} u_{i j}(x) \eta_{i} \eta_{j} \leq \sum_{i, j=1}^{2 N} \phi_{i j}(x) \eta_{i} \eta_{j} .
$$

By (4) with $\xi=D \phi(x)$ and $X=D^{2} \phi(x)$ we find

$$
F\left(D \phi(x), D^{2} \phi(x)\right)=\frac{1+\left(\phi_{n}(x)\right)^{2}}{2^{3}} \sum_{j=1}^{2 N} \phi_{j j}(x) \geq 0,
$$

and we can conclude that $u$ is a viscosity subsolution of $F=0$ at $x \in \Omega$. 
Analogously, for every $\phi \in C^{2}(\Omega)$ such that $u-\phi$ has a local minimum at $x$, we have $\phi_{j}(x)=u_{j}(x)=0$ for every $j=1, \ldots, 2 N$, and for every $\eta \in \mathbb{R}^{2 N}$

$$
0=\sum_{i, j=1}^{2 N} u_{i j}(x) \eta_{i} \eta_{j} \geq \sum_{i, j=1}^{2 N} \phi_{i j}(x) \eta_{i} \eta_{j}
$$

then

$$
F\left(D \phi(x), D^{2} \phi(x)\right)=\frac{1+\left(\phi_{n}(x)\right)^{2}}{2^{3}} \sum_{j=1}^{2 N} \phi_{j j}(x) \leq 0,
$$

and we can conclude that $u$ is a viscosity supersolution of $F=0$ at $x \in \Omega$.

Thus, $u$ is a viscosity solution of $F=0$ in $\Omega$.

\section{References}

[1] E. BEDFORD and B. GAVEAU, Hypersurfaces with Bounded Levi Form, Indiana University Journal 27(5) (1978), 867-873.

[2] S. BERNSTEIN, Sur la généralisation du problème de Dirichlet II, Math. Ann. 69 (1910), 82-136.

[3] S. BERNSTEIN, Sur les équations du calcul des variations, Ann. Sci. École Norm. Sup. 29 (1912), 431-485.

[4] E. BOMBIERI, E. DE GIORGI and M. MIRANDA, Una maggiorazione a priori relativa alle ipersuperfici minimali non parametriche, Arch. Rational Mech. Anal. 32 (1969), 255-267.

[5] G. CITTI, E. LANCONELLI and A. MONTANARI, Smoothness of Lipschitz continuous graphs with non vanishing Levi curvature, Acta Mathematica 188 (2002), 87-128.

[6] A. DEBIARD and B. GAVEAU, Problème de Dirichlet pour l'équation de Lévi, Bull. Sci. Math. (2) 102(4) (1978), 369-386.

[7] H. FEDERER, Geometric measure theory, Berlin-Heidelberg-New York, Springer Verlag, 1969.

[8] R. FINN, On equations of minimal surface type, Ann. of Math. 60(2) (1954), 397-416.

[9] D. GILBARG and N. S. TRUDINGER, Elliptic partial differential equations of second order, Grundlehrer der Math. Wiss. 224, Springer-Verlag, New York, 1977. 
[10] O. A. LADYZHENSKAYA, Solution of the first boundary problem in the large for quasi-linear parabolic equations, Trudy Moscov Mat. OBšč 7 (1958), 149-177 [Russian].

[11] O. A. LADYZHENSKAYA and N. N. URAL'TSEVA, Quasilinear elliptic equations and variational problems with several indipendent variables. Uspehi Mat. Nauk 16(1) (1961), 19-90. [Russian]. English translation in Russian Math. Surveys 16(1) (1961), 17-92.

[12] O. A. LADYZHENSKAYA and N. N. URAL'TSEVA, Linear and quasilinear Elliptic Equations Moskow: Izdat. Nauka 1964 [Russian]. English Translation: New York, Academic Press, 1968. 2nd Russian ed. 1973.

[13] O. A. LADYZHENSKAYA and N. N. URAL'TSEVA, Local estimates for gradient of solutions on non-uniformly elliptic equations, Comm. Pure Appl. Math. 23 (1970), 677-703.

[14] V. MARTINO and A. MONTANARI, Graphs with prescribed Levi form trace, Annali dell'Università di Ferrara Sezione VII 52 (2006), 371-382.

[15] A. MONTANARI and E. LANCONELLI, Pseudoconvex fully nonlinear partial differential operators. Strong comparison Theorems, J. Differential Equations 202(2) (2004), 306-331.

[16] J. SERRIN, Gradient estimates for solutions of nonlinear elliptic and parabolic equations, In: Contributions to Nonlinear Functional Analysis, New York, Academic Press, 1971, 565-601.

[17] Z. SLODKOWSKI and G. TOMASSINI, Weak solutions for the Levi equation and envelope of holomorphy, J. Funct. Anal. 101(2) (1991), 392-407.

Received 31 May 2006; accepted 25 July 2006

To access this journal online:

http://www.birkhauser.ch 